PSU-TH-209

hep-th/9906238

\title{
Ultraviolet Limit of Open String Theory
}

\author{
Shyamoli Chaudhuri ${ }^{1}$ \\ Physics Department \\ Penn State University \\ University Park, PA 16802
}

\begin{abstract}
We confirm the intuition that a string theory which is perturbatively infrared finite is automatically perturbatively ultraviolet finite. Our derivation based on the asymptotics of the Selberg trace formula for the Greens function on a Riemann surface holds for both open and closed string amplitudes and is independent of modular invariance and supersymmetry. The mass scale for the open strings stretched between Dbranes suggests a natural world-sheet ultraviolet regulator in the string path integral, preserving both T-duality and open-closed string world-sheet duality. Note added (Jan 2005): Comments and related references added.
\end{abstract}

\footnotetext{
${ }^{1}$ Current Address: 1312 Oak Dr, Blacksburg, VA 24060. shyamolic@yahoo.com
} 
In developing a formalism for nonperturbative string theory, it is important to clarify which properties of the theory follow from world-sheet principles alone. Finiteness in the ultraviolet limit is a fundamental property of the perturbative amplitudes of an infrared finite string theory, well known to string experts [1]. Unfortunately, since most discussions of finiteness are carried out in the context of superstring theory it is often incorrectly attributed to supersymmetry. It has also been misconstrued as a consequence of modular invariance - a property of only the closed string sector of the theory. To clarify both of these points unambiguously, we will examine the worldsheet ultraviolet limit of the amplitudes of the oriented bosonic string with multiple boundaries on Dbranes. Parallel and static Dpbranes separated by a distance $r$ in a direction orthogonal to the branevolume lead to BPS configurations in string theory: left-moving string modes are identified with right, upon spacetime reflection at either Dbrane. In the superstring this breaks half of the spacetime supersymmetries. Relative motion of the branes in a direction orthogonal to the branevolume or, equivalently, a relative spatial rotation of the branes in a plane embedded in the transverse space orthogonal to the branevolume, breaks further the spacetime supersymmetries. But, as we will see, the absence of infinities in the ultraviolet limit for an infrared finite string theory are quite independent of such supersymmetry breaking boundary conditions.

Infrared finite string theories require the absence of tachyon and tadpole states in the string spectrum [1]. These conditions are easily achieved by, respectively, introducing fermionic degrees of freedom on the world-sheet, and including nonorientable world-sheets in the path integral. The effect of the negative mass tachyon and the zero momentum massless states, or tadpoles, that characterize the infrared limit of the oriented bosonic string can be exposed explicitly in the path integral, distinguishable from other features of the amplitude generic to any string theory. Our derivation of the worldsheet ultraviolet asymptotics of the bosonic string path integral, based on the Selberg trace formula [2], can be applied to any string amplitude, open or closed, and rests upon open-closed string world-sheet duality alone. The result can in fact be inferred from previous work on bosonic closed string amplitudes $[3,4,25,26]$. We have simply clarified their extension to both open and closed string amplitudes.

Unlike amplitudes in the closed string sector, where an infrared divergence 
can always be mapped to ultraviolet and vice versa by a symmetry of the amplitude - modular invariance, it is meaningful to distinguish the infrared and ultraviolet limits of the open string sector. In an open and closed string theory, open-closed worldsheet duality instead maps the infrared behavior of the open string spectrum to the ultraviolet behavior of the closed string spectrum [1,37]. Let us review this point with a discussion of the annulus diagram in bosonic string theory. Parameterize the annulus with world-sheet coordinates $0 \leq \sigma^{1} \leq 1,0 \leq \sigma^{2} \leq 1$, and boundaries of length $l$. The tree exchange of oriented closed strings between a pair of parallel Dpbranes with separation $r$ in a direction, $X^{24}$, and relative velocity $v$ in a direction, $X^{25}$, can be written in the form $[28,29]$ :

$$
\mathcal{A}(r, v)=V_{26} \int_{0}^{\infty} \frac{d l}{l}\left(8 \pi^{2} \alpha^{\prime} l\right)^{-p / 2} e^{-r^{2} l / 2 \pi \alpha^{\prime}}\left[l^{-1 / 2} \eta(i / l)\right]^{-22}\left[\frac{\eta(i / l)}{-\Theta_{11}(-i u / \pi, i / l)}\right]
$$

where $v=\tanh u$. Small $r$ corresponds to large $l$ or, in terms of the inverse length parameter, $\tilde{l}=1 / l$, small $\tilde{l}$. The closed string tachyon and "wouldbe" dilaton tadpoles present in the $r \rightarrow \infty$ infrared limit are exposed by expanding the integrand in powers of $e^{-2 \pi \tilde{l}}$ :

$$
\begin{array}{ll}
\lim _{\tilde{l} \rightarrow \infty} \quad \tilde{l}^{(p-24) / 2} e^{-r^{2} / 2 \pi \alpha^{\prime} \tilde{l}} \frac{e^{2 \pi \tilde{l}}}{2 i \operatorname{Sinh}(u)} \\
\quad \times\left[1+e^{-2 \pi \tilde{l}}(22+2 \operatorname{Cosh}(2 u))+O\left(e^{-4 \pi \tilde{l}}\right)\right] .
\end{array}
$$

The leading term is absent in a tachyon-free infrared finite string theory. Note that the tension of the stretched string, $M_{W} \sim r^{2} l / 2 \pi \alpha^{\prime}$, gives a natural cutoff on the $l \rightarrow 0$ limit of the string path integral.

Conversely, we can expand in powers of $e^{-2 \pi l}$, which exposes the $r \rightarrow 0$ ultraviolet limit of the amplitude:

$$
\begin{aligned}
\lim _{l \rightarrow \infty} \quad & l^{-(p+2) / 2} e^{-r^{2} l / 2 \pi \alpha^{\prime}} \frac{e^{-u^{2} l / \pi}}{2 i \operatorname{Sin}(u l)} e^{2 \pi l} \\
& \times\left[1+e^{-2 \pi l}(22+2 \operatorname{Cos}(2 u l))+O\left(e^{-4 \pi l}\right)\right] .
\end{aligned}
$$

In the absence of the leading term in the expansion, in an infrared finite string theory, the ultraviolet limit will be automatically finite quite independent of spacetime supersymmetry. The ultraviolet limit is then an open string 
abelian gauge theory with the Goldstone boson that arises from the spontaneous breaking of spacetime translational invariance by Dbrane boundaries appearing at the first massive level in the open string spectrum $[6,7]$. The mass of the stretched string plays the role of the classical mass of the Goldstone boson. We will explore the analogous limit of the tree exchange amplitude of multiple bosonic Dbranes [28], using the Selberg trace formalism $[2]$.

The theta functions in Eq. (1) are the result of summing over eigenmodes of the Laplacian on the annulus with $p$ Neumann and $24-p$ Dirichlet coordinates, and the boundary conditions: $X^{25}=0, \partial_{2} X^{0}=0$ at $\sigma^{2}=0$, and $X^{25}=v X^{0}$ at $\sigma^{2}=1$, on coordinates $X^{25}, X^{0}$. An intuitive picture of the large/small $l$ asymptotics is provided by the diffusion time of a given eigenmode on the world sheet $[9,8]$. The Greens function of the diffusion operator, $\partial_{t}-\Delta$, is a trace over the eigenvalue spectrum, $\lambda_{\left(m_{1}, m_{2}\right)}=4 \pi^{2}\left(m_{1}^{2}+m_{2}^{2} \tilde{l}^{2}\right)+E_{\text {vac. }}(u)$, and $-\infty \leq m_{1} \leq \infty, m_{2}>0$ :

$$
\ln \operatorname{det}^{\prime} \Delta=-\int_{0}^{\infty} d t t^{-1} \int d^{2} z \sqrt{g} G\left(z, z^{\prime} ; t\right) .
$$

Symmetrizing under $m_{2} \rightarrow-m_{2}$, a Poisson resummation expresses the trace in the equivalent form:

$$
\begin{aligned}
\sum_{m_{1}, m_{2}} & e^{-4 \pi^{2}\left(m_{1}^{2}+m_{2}^{2} \tilde{l}^{2}\right) t-2 m_{2} u} \\
& =\sum_{n_{1}, n_{2}} \frac{A}{(4 \pi t)^{1 / 2}} e^{-\left(n_{1}^{2}+\left(n_{2}-i u / \pi\right)^{2} l^{2}\right) / 4 t},
\end{aligned}
$$

where $A \equiv l$ is the area of the surface. For small diffusion times only the lowest lying eigenmode is excited; this is the small $l$ limit which, due to the stretched string background, is the same as the large $r$ limit. For large diffusion times, several modes can be excited together. The dominant contribution to the path integral comes from the most excited states: heavy closed strings, or, by world sheet duality, light open strings [7].

With this picture in mind, we now consider the extension to open string amplitudes with $b \geq 3$ boundaries on Dbranes. If we wish to identify the small $l$ limit of this amplitude as the tree gravitational interaction of $b$ Dbranes with separations of order $r>>\sqrt{\alpha}$, the boundaries live on distinct branes. Now the expression on the right hand side of Eq. (5) has a simple geometric 
interpretation. An arbitrary surface with cylindrical topology maps to a rectangular cell in the complex plane with edges of metric length $(1, l)$. Thus the eigenvalue trace is a sum over a length spectrum of geodesic paths: paths joining opposite edges of the unit cell, or, by the translational symmetry, $\operatorname{Re} z \rightarrow \operatorname{Re} z+1$, the opposite edges of two cells related by $n_{1}$ translations. An extension of this idea underlies the Selberg trace formula for the Greens function of the diffusion operator on a Riemann surface, $S$, with $b \geq 3$ boundaries $[2,8]$.

Since the sums over eigenfunctions are potentially divergent we introduce regulators. Consider the expression:

$$
\operatorname{lndet}^{\prime} \Delta=-\lim _{s \rightarrow 0} \frac{d}{d s} \int_{\epsilon}^{\infty} \frac{d t t^{s-1}}{\Gamma(s)} \int_{S} d^{2} z \sqrt{g}\left[\operatorname{tr}^{\prime} e^{-t\left(\Delta+m^{2}\right)}\right]-e^{-m^{2} t},
$$

where $\epsilon$ and $m^{2}$ are, respectively, world-sheet ultraviolet, and infrared, regulators, the latter being necessary only in cases where the Laplacian develops a zero mode. The infrared limit is then regulated by Pauli Villars subtraction as in $[3,4,37]$. We will denote the factor in square brackets as $\hat{G}\left(z, z^{\prime} ; t\right)$. Notice that the introduction of a cutoff on diffusion time gives an ultraviolet regulator formally violates worldsheet Weyl invariance, introducing an explicit mass scale, $M_{W}$, into the path integral formalism [37]. $M_{W}$ behaves like an ultraviolet cutoff from the perspective of the worldvolume effective gauge theory. However, this prescription preserves both $\mathrm{T}$-duality, which relates open string backgrounds with distinct numbers of Dirichlet/Neumann coordinates, and open-closed string world sheet duality [1]. It should be distinguished from the finite world sheet cutoff for general nonperturbative string backgrounds proposed in [10], which would break world sheet duality explicitly.

The Greens function, $\hat{G}\left(z, z^{\prime} ; t\right)$, is assembled from the basis of eigenfunctions of the Laplacian on the Riemann surface $S_{b}$ with Euler character $\chi=2-b$. The divergent contribution from the Greens function at the source, $\hat{G}(z, z ; t)$, will be understood to have been subtracted from this trace; it can be evaluated by the same method. By the uniformization theorem $[11,12]$, we can map such a surface to a polygon $H / \Gamma_{b}$, a $-9 \chi$-sided subdomain of the upper half complex plane, $H$, with constant negative curvature, $R_{g}=-1$, area element, $d A=d x d y / y^{2}, y>0$, and, from the Gauss-Bonnet theorem, constant area, $A=-4 \pi \chi . \Gamma_{b}$ is the fundamental, or covering, group of the 
Riemann surface. The Laplacian takes the form $\Delta=y^{-2}\left(\partial_{x}^{2}+\partial_{y}^{2}\right)$. By the Riemann-Roch theorem, the shape of the surface is characterized by $-3 \chi$ real parameters. We choose these to be the Fricke-Klein moduli $[12,13,14]$ : the lengths of $b$ holes, and $2(b-3)$ interior paths on $S_{b}$, closed and nonintersecting, and geodesic with respect to the Poincare line element, $d s^{2}=d \rho^{2}+\operatorname{Sinh}^{2} \rho d \theta^{2}$. These will be described in an example below. See, also, the discussion of uniformization and moduli in the appendix. Thus, the length of a geodesic path on $H$ through the points $z, z^{\prime}$ is given by the minimum:

$$
\rho_{H}\left(z, z^{\prime}\right)=\min \int_{C} \frac{d y}{y}=\operatorname{minCosh}^{-1}\left[1+\frac{\left|z-z^{\prime}\right|^{2}}{2 y y^{\prime}}\right]^{1 / 2}
$$

where $C$ is an arbitrary path on $H$ linking $z, z^{\prime}$.

Thus, $\hat{G}$ on $S_{b}$ is composed from the basis of eigenfunctions of the Laplacian on the Poincare upper half plane, $H$, with the periodicity property, $\Psi(x+\tilde{x}, y ; q)=e^{2 \pi i q \tilde{x}} \Psi(x, y ; q)$, under shifts, $\operatorname{Re} z \rightarrow \operatorname{Re} z+\tilde{x}$, where $q$ is a real parameter. For eigenvalues parameterized as $\lambda=-\left(\frac{1}{4}+v^{2}\right)$, with $v$ real, these take the form [22]:

$$
\Psi_{(q, \lambda)}=N_{(q, v)} y^{1 / 2} K_{i v}(2 \pi|q| y) e^{2 \pi i q x},
$$

where $K_{i v}(w)$ is a modified Bessel function. Neumann/Dirichlet boundary conditions may be imposed by evaluating the Greens function on the double of $S_{b}$, a closed orientable Riemann surface with $b-1$ handles, and restricting to the subspace of eigenfunctions that are, respectively, even/odd with respect to reflection in the boundary [22].

The normalization, $N_{(q, v)}$, is determined by the integration measure:

$$
\hat{G}\left(z, z^{\prime} ; t\right)=N_{(q, v)}^{2} \int_{-\infty}^{\infty} d q \int_{-\infty}^{\infty}[v \operatorname{Sinh}(\pi v) d v] \sqrt{y} \sqrt{y^{\prime}} K_{i v}(2 \pi|q| y) K_{i v}\left(2 \pi|q| y^{\prime}\right)
$$

invariant under linear fractional transformations $z^{\prime} \equiv \gamma[z]=\frac{a z+b}{c z+d}$ with $a, b, c, d$ real, and $a d-b c=1$, that take the Poincare upper half plane into itself. Thus, $\gamma$ is an element of the group $P S L(2 ; R)$, and the factor in square brackets can be recognized as the group invariant measure. Integration over the "angular "variable $2 \pi q$ gives the associated Legendre function, $P_{i v-\frac{1}{2}}\left[\operatorname{Cosh}\left(\rho_{H}\left(z, z^{\prime}\right)\right)\right]$. Following some simple manipulations [22], the Greens function $\hat{G}$ on $H$ can 
be put in the form:

$$
\hat{G}\left(z, z^{\prime} ; t\right)=\frac{1}{(4 \pi t)^{3 / 2}} \int_{\rho_{H}}^{\infty} \frac{\sqrt{2} b e^{-b^{2} / 4 t-M^{2} t}}{\sqrt{\operatorname{Cosh} b-\operatorname{Cosh} \rho_{H}}} d b,
$$

where $\rho_{H}$ is the length of the geodesic path joining points $z, z^{\prime}$, and the integral sums over lengths, $b$, of arbitrary paths on $H$ joining $z, z^{\prime}$. The parameter $M^{2}=m^{2}+\frac{1}{4}$. The Greens function for coincident points $z^{\prime}=z$ is obtained by simply setting the argument of the Greens function to the ultraviolet cutoff on the world-sheet, a parameter of $O(\epsilon)$. Insight into the physical meaning of $\epsilon$ will be obtained by considering the full amplitude as shown below.

The precise spectrum of eigenvalues of $\Delta$ on $H$ is not known by analytic means [22]. However the growth of the density of eigenvalues for large $\lambda$ can be estimated. We will use this estimate below to bound the behavior of the Greens function on $S_{b}$ for large $\lambda$. The opposite limit of small eigenvalues, and short geodesics, determines the $l \rightarrow 0$ limit of the amplitude $[25,26]$. The Selberg trace formula expresses the Greens function on the fundamental polygon $H / \Gamma_{b}$ to that on the covering space $H[2,8]$. Some key steps are reviewed in the appendix. The fundamental group $\Gamma_{b}$ of $S_{b}$ is the $-3 \chi$ parameter Fuchsian subgroup of linear fractional transformations: every element is related by a similarity transformation to a magnification, $z^{\prime}=\alpha[z]$, with generating elements constrained by the relation $\prod_{i=1}^{3(b-2)} \gamma_{i}=\mathbf{1}$, and $\operatorname{tr} \gamma>2$ for all $\gamma \in \Gamma_{b}$. More details can be found in the appendix. The magnification, $z^{\prime}=\alpha[z]$, defines a geodesic path on $H / \Gamma_{b}$, with length, $\rho_{H}\left(z, z^{\prime}\right)$, defined as above, and the $-3 \chi$ real parameters of the generating group elements can be explicitly related to the Fricke-Klein moduli of $S$ as shown in [13, 14, 15].

To gain some intuition into the moduli, consider orientable surfaces with four holes, $C[i]$, of common length of $O(l)$, and two interior geodesics, homotopic to $C[1] C[2], C[2] C[3]$, of length $l_{S}, l_{T}$. The reader may find it helpful to keep in mind the bosonic contribution to the tree scattering amplitude of four Dobranes in type IIA string theory. The simpler $b=3$ pants amplitude does not exemplify the general case since it lacks interior moduli. Let the distances between pairs of Dbranes satisfy the inequalities, $r_{12} \sim r_{34} \geq \sqrt{\alpha^{\prime}}$, with $r_{13} \sim r_{14} \sim r>>>r_{12}$. Then the $l \rightarrow 0$ limit of the amplitude is dominated by world-sheets with four long tubes of length $\tilde{l}$ attached to the respective Dbranes. The remaining two moduli, $l_{S}, l_{T}$, can be interpreted 
as the "proper time" for propagation of an intermediate closed string state in, respectively, the $\mathrm{S}$ and $\mathrm{T}$ channels of the amputated amplitude. An explicit representation of the generating elements of the group $\Gamma_{4}$ in terms of $\operatorname{PSL}(2, R)$ matrices, and the parameterization in terms of the six Fricke-Klein moduli of $S_{4}$, is given in [14].

Alternatively, one can use the Fenchel-Nielsen length-twist parameterization of the interior geodesic moduli $[17,14,20]$. Returning to the example above, a pair of coordinates, $l_{S}, \theta_{S}$, where $\theta_{S}$ is the Dehn twist of $C[1] C[2]$ relative to $C[3] C[4]$ before identification. In the limit $l \rightarrow 0, l_{S} \rightarrow 0$, the static amplitude factorizes into the product of four disc tadpole amplitudes with emanating closed string propagators, and a closed string propagator linking two cubic closed string interaction vertices, spatially separated by a distance of $O(r)$. Upon setting the branes in motion, we can extract from this amplitude an $O\left(g_{c}^{2}\right)$ correction to the bosonic sector of an eight-point function of supergravitons in M theory [16]. Here, $g_{c}$ is the closed string coupling.

Note that the presence of classical mass terms for the Goldstone bosons in the string path integral - in this example, $M_{W} \sim O\left(r / \alpha^{\prime}\right)$, with $r_{13} \sim$ $r_{14}>>>r_{12} \sim r_{34}$, implies a lower cutoff, $l_{\text {min. }}$, on the integrals over geodesic loop lengths. Let $l_{\text {min. }}$ be the shortest, non-contractible, geodesic loop length summed in the string path integral, a parameter of $O\left(\alpha^{\prime} / r^{2}\right)$. Note that cutting off the integrals over geodesic loop length at $l_{\min }>0$ amounts to a formal violation of Weyl invariance, and can be interpreted as the introduction of a world-sheet ultraviolet regulator, $\epsilon$, of $O\left(\alpha^{\prime} / r^{2}\right)$. This prescription can be compared with the observation in [21], where the role of the "W" boson mass as a spacetime infrared regulator in the bulk supergravity theory was noted. This is a consequence of the stretched string backgrounds, since small $l$ is the same as large $r$ in the presence of the stretched strings. With the FenchelNielsen coordinates as parameterization of conformally inequivalent classes of world sheet metrics, $\mathcal{T} \equiv \mathcal{M}_{g} /$ Conf $\times$ Diff $_{0}$, the measure in the path integral takes the simple form $[31,28]$ :

$$
\begin{aligned}
\mathcal{A}=\quad & V_{26} A^{(-3 \chi+p) / 2}\left(4 \pi^{2} \alpha^{\prime}\right)^{-p / 2} \int \prod_{m=1}^{2 b-3} \prod_{n=1}^{b-3} d l_{m} d \theta_{n} \\
& \times e^{-\sum_{i \neq j} \frac{1}{2 \pi \alpha^{\prime}} r_{i j}^{2} l_{j}}\left(\operatorname{Det}^{\prime} \Delta_{1}\right)^{1 / 2}\left(\operatorname{Det}^{\prime} \Delta_{0}^{(26)}\right)^{-1 / 2},
\end{aligned}
$$

where the normalization of the amplitude is understood to be absorbed in the regulated spacetime volume, $V_{26}$, and the functional determinants are to be 
computed with the given boundary conditions by an extension of the method below [4, 31]. An arbitrary metric in $\mathcal{M}_{g}$ can be uniquely transformed by a conformal transformation plus diffeomorphism continuously connected to the identity, to the fiducial constant curvature metric $g[18,19,4]$. The parameterization of $\mathcal{T}$ is not unique. For a different parameterization, the measure in the path integral is given by the determinant computed in [3]:

$$
\prod_{i=1}^{-3 \chi} d \tau_{i}\left[\operatorname{det}\left(\left(\chi_{k}\right)_{e f}\left(\chi_{l}\right)^{e f}\right)\right]^{1 / 2}
$$

where $\chi_{k}^{a b} \equiv \partial_{k} g^{a b}-\frac{1}{2} g^{a b} g^{c d} \partial_{k} g_{c d}$, and $g_{a b}$ is the fiducial metric on some gauge slice in the space of metrics, $\mathcal{M}_{g}$. The Fenchel-Nielsen coordinates for $\mathcal{T}$ correspond to the choice for which this determinant is trivial. The moduli dependence is then entirely contained in the lengths, $l_{i}$, of $2 b-3$ closed geodesic paths on $S[14,15], b-3$ of which are interior paths, and $b-3$ corresponding twist angles, $\theta_{j}[17,20,31]$. Explicit examples and more details can be found in [14].

The Greens function on the polygon $H / \Gamma_{b}$ can be written as:

$$
\operatorname{tr}^{\prime} e^{-\left(\Delta+m^{2}\right) t}=\sum_{\gamma \neq \mathbf{1}} \int_{H / \Gamma_{b}} \frac{d x d y}{y^{2}} \hat{G}(z, \gamma[z] ; t)+A(H / \Gamma) \cdot \hat{G}(z, z ; t) .
$$

The identity element in the fundamental group, $\gamma[z]=z$, gives the Greens function at the source, $\hat{G}(z, z ; t)$. It has been separated from the sum over group elements in Eq. (13). This defines the Poincare theta series, $\Theta(t)$, described briefly in the appendix [8]. Consider the primitive elements of $\Gamma_{b},\{\delta\}$, transformations which cannot be expressed as a power of any other transformation. Then an arbitrary $\gamma \in \Gamma_{b}$ can always be related by a similarity transformation to an integer power of a primitive element, $\gamma=\beta \delta^{p} \beta^{-1}$, for suitable integer $p$, and element $\beta$ noncommuting with $\delta$. For surfaces with $b$ holes, such a similarity transformation can be used to bring any primitive element $\delta$ to the form of a magnification $[2,8]$. It follows that the length parameter of the element, $\delta^{p}$, satisfies the relation $l\left(\delta^{p}\right)=p l(\delta)$. The use of the similarity transformations then allow us to derive $[2,8]$ :

$$
\operatorname{lndet}^{\prime} \Delta \equiv \quad-\lim _{s \rightarrow 0} \frac{d}{d s}\left[\frac{1}{\Gamma(s)} \int_{\epsilon}^{\infty} d t t^{s-1}\left[\Theta(t)+A \cdot \hat{G}\left(\operatorname{Cosh}\left(l_{\text {min. }}\right) ; t\right)\right]\right]
$$




$$
=\quad-\lim _{s \rightarrow 0} \frac{d}{d s}\left[\sum_{\{\delta\}} \sum_{p=1}^{\infty} \frac{l(\delta) / 2}{\operatorname{Sinh}(p l(\delta) / 2)} \int_{\epsilon}^{\infty} \frac{d t t^{s-3 / 2}}{\Gamma(s) \sqrt{\pi}} e^{-p^{2} l(\delta)^{2} / 4 t-M^{2} t}\right] .
$$

Some key steps are given in the appendix. The factor in square brackets is a generalized zeta function $\zeta_{R}(s)[2,8,27]$. Note that the integral over diffusion times, which we will denote by $I\left(p l(\delta) ; \epsilon, m^{2}\right)$, is regulator dependent.

The sum over primitive elements in Eq. (14) needs further comment. The spectrum of geodesic lengths on $S_{b}$ is discrete, a direct consequence of the discreteness of the eigenvalue spectrum of the Laplacian [22]. The $l \rightarrow 0$ limit determines the infrared $r \rightarrow \infty$ behavior of the amplitudes and has been extensively studied in the literature $[25,26]$. Consider the double of the Riemann surface, $S_{b}$, a closed oriented surface with $b-1$ handles and $2(b-3)+b$ pairs of Fenchel-Nielsen moduli: $l_{j}, \theta_{j}, j=1, \cdots, 3(b-2)$. In the limit of a pinched cycle, $l_{j} \rightarrow 0$ for some $j$, the world-sheet develops a long tubular neck, or collar, with hyperbolic metric and eigenvalue spectrum, determined by the behavior of the minimum length core geodesic on the pinched cycle [25]. The length and twist moduli of the pinched cycle become redundant. The eigenvalue spectrum on the complement of the surface, with collar region excised, can then be bounded, and the divergence in the spectrum of the collar component isolated. The result is similar to the long cylinder limit described earlier: in either case, it comes from the clustering of low frequency, rotationally invariant, eigenmodes at the limit point: $\lambda=-\frac{1}{4}$ [25]. This analysis of the $l \rightarrow 0$ asymptotics of the trace formula can be modified in the presence of a finite cutoff on the world-sheet. Some preliminary steps are given in the appendix.

We will consider here the opposite limit of the amplitude, the worldsheet infrared $l \rightarrow \infty$ regime, dominated by the behavior of the large eigenvalues, or long geodesics. We will find that our suggested introduction of an ultraviolet cutoff $\epsilon$ on the $l$ integral will result in an $\epsilon \sim O\left(\alpha^{\prime} / r^{2}\right)$ dependent correction to the leading behavior of $\zeta_{R}(s)$ in this limit. We find, however, that this correction is exponentially damped as $\epsilon \rightarrow 0$, confirming the intuition that a perturbatively infrared finite string theory is automatically perturbatively ultraviolet finite when worldsheet Weyl invariance has not been violated.

Cut off the sum over primitives for the first $N(L)$ primitives, partitioned among $M$ distinct lengths, $l_{m}, m=1 \cdots M$, upto some maximum value $L$, with degeneracies $n_{m}, \sum_{m=1}^{M} n_{m}=N$. For sufficiently large $M, L$, the sequence of lengths converges to a continuum and we can replace the factor 
in square brackets in Eq. (14) with an integral over the density of lengths $[24,8]$ :

$$
\begin{aligned}
& \lim _{L \rightarrow \infty}\left[\sum_{\{\delta ; l(\delta)<L\}} \cdots\right]=\frac{1}{\Gamma(s)} \sum_{p=1}^{\infty} \\
& \times \int_{l_{\min } .}^{L} d \ln (l) 2 l e^{-p l / 2}\left(1-e^{-p l}\right)^{-1} I\left(p l ; \epsilon, m^{2}\right) .
\end{aligned}
$$

The growth in the degeneracy of primitive elements of fixed length $L$ is known from Huber's estimate [24, 8]:

$$
\lim _{L \rightarrow \infty} \int_{l_{\min }}^{L} n(l) d l=(1+\alpha) \frac{1}{L} e^{L}
$$

where $\alpha$ is a constant of order less than one. Interchanging the order of integration over $t, l$ and substituting from Eq. (16), we see that the $p=1$ term in the sum dominates the bound. Thus,

$$
\lim _{L \rightarrow \infty} \zeta_{R}(s) \leq \frac{1}{\Gamma(s)} L e^{-L / 2} \cdot \frac{1}{L} e^{L} \cdot I\left(L ; \epsilon, m^{2}\right) .
$$

The integral over $t$ can be bounded for large $L$ by splitting the range of integration, $\int_{\epsilon}^{\infty}=\int_{0}^{\infty}-\int_{0}^{\epsilon}$, and estimating the correction to the resulting modified Bessel function from the short time behavior of $I\left(L ; \epsilon, m^{2}\right)$. The result is the bound:

$$
\lim _{L \rightarrow \infty} \zeta_{R}(s) \leq \frac{e^{L / 2}}{\Gamma(s)}\left[L^{\nu-1 / 2} e^{-L / 2}+O\left(\epsilon^{\nu+1} L^{-2} e^{-L^{2} / 4 \epsilon}\right)\right],
$$

where $\nu=s-1 / 2$. The leading behavior can already be extracted from [4]. We have simply clarified its extension to the Greens function on surfaces with boundaries. As expected, for large $L$, the $\epsilon$ dependent correction is insignificant unless $r<<\sqrt{\alpha^{\prime}}$. Similar estimates can be made for the Greens function at the source, $G\left(\operatorname{Cosh}\left(l_{\min } ; \epsilon\right)\right)$, and the Greens functions of the Laplace-Beltrami operators for higher rank tensor fields on $S_{b}[30,4,25]$. These include the vector Laplacian that appears in the measure for the string path integral. The functional methods described here can be extended to amplitudes with general boundary conditions [1]: moving [29], mixed [32]for configurations with Dbranes of different dimensionality, external field and 
finite temperature [33], and, most importantly, supersymmetric. Work in this direction is in progress.

Thus far, we have restricted ourselves to a discussion of the open string amplitude with multiple boundaries on separated Dbranes, $r_{i j}>\sqrt{\alpha^{\prime}}$, for every $i, j=1, \cdots, b$. Consider the configuration with $N$ superimposed Dbranes, within distances of $O\left(\sqrt{\alpha^{\prime}}\right)$, and a single Dbrane at a distance $r>>\sqrt{\alpha^{\prime}}$. The $l \rightarrow \infty$ asymptotics of the string path integral yields the behavior of a nonabelian, $U(N)$, gauge theory on the Dbrane stack [1].

We conclude with the observation that modifying the Weyl-covariant open string perturbation theory in stretched string backgrounds by introduction of a finite world-sheet ultraviolet cutoff, $\epsilon \simeq \alpha^{1 / 2} / r$, where $r$ is the length of the stretched string, can indeed give a prescription that preserves both open-closed world-sheet duality, and the target space T-dualities. More importantly, it might also provide a quantitative description of the crossover regime:

$$
N^{1 / 7} M_{P}^{-1}<r<N^{1 / 3} M_{P}^{-1},
$$

between supergravity and perturbative super Yang-Mills theory [35, 16, 36]. Here, $M_{P}^{-1}=g_{c}^{1 / 3} \sqrt{\alpha^{\prime}}$, is the eleven dimensional Planck length, and $r$ has been identified with the typical length scale probed by the dynamics, i.e., the spatial separation of two point sources in 11-d supergravity. This corresponds to Dobrane dynamics in the type IIA string theory or, by a T-duality in the direction $X^{9}$, to D1brane dynamics in the type IIB string theory. The upper bound in Eq. (19) is the t'Hooft radius, marking the transition to perturbative SYM theory. The lower bound, where the closed string coupling $g_{c} \sim O(1)$, is the transition to the low energy limit of $\mathrm{M}$ theory. But it should be emphasized that, as a consequence of our having explictly broken worldsheet Weyl invariance, this prescription is only effective: the renormalizability of the low energy gauge theory limit in an exactly solvable conformal field theory background of an infrared finite perturbative string theory, even in the absence of target spacetime supersymmetry [38], has been obscured $[37]$.

What is the significance of a world-sheet ultraviolet regulator that preserves both open-closed world-sheet duality, and T-duality, although it violates the Weyl invariance of the covariant worldsheet formalism? Our conjecture is that IIA string perturbation theory with finite ultraviolet cutoff 
$\epsilon \sim O\left(\alpha^{\prime} / r^{2}\right)$ might fill the gap between the kinematic regimes of DLCQ, the discrete light cone quantization of $\mathrm{M}$ theory, and of 11-d supergravity $[16,36]$. Upon T-dualizing, this would cover the crossover region between the supergravity and perturbative SYM regimes of the IIB string theory [34, 35]. Whether this can lead to detailed comparisons between the modified open string perturbation theory, and nonperturbative formulations of DLCQ [36], remains to be investigated in the future.

\section{Acknowledgments}

I would like to thank J. Polchinski for inspiration. I also thank the Institute for Theoretical Physics and the Aspen Center for Physics for their hospitality. I am grateful to E. D'Hoker for providing me with references and comments on previous work. Finally, I must thank my students E. Novak and Y. Chen for their questions during the course of this work. This research is supported in part by NSF grant PHY 97-22394.

Note added (Jan 2005): Minor corrections in wording have been made in the text of the paper to clarify that the introduction of an explicit cutoff on the boundary loop lengths, as might seem natural in the background of stretched strings, amounts to a formal violation of worldsheet Weyl invariance [37]. We emphasize that the conclusion that a perturbatively infrared finite string theory is automatically ultraviolet finite, even in the absence of target spacetime supersymmetry [38, 39], holds only in the limit of unbroken Weyl invariance: $\epsilon \rightarrow 0$ [37]. A consequence of broken Weyl invariance is that the worldvolume gauge theory obtained in the low energy limit of the string theory is only a Wilsonian effective field theory, with ultraviolet (uv) cutoff provided by the tension of the stretched string. From the perspective of the gauge theory, the uv cutoff can be interpreted as the mass of the Goldstone boson associated with the spontaneous breaking of Poincare invariance in the bulk target spacetime by the Dbranes. Refs. [37, 38, 39] are new.

\section{A The Selberg Trace Formula}

The basic observation underlying the derivation of Selberg's trace formula is as follows [2]. A readable introduction appears in [8]. The Greens function 
of the scalar Laplacian on $S$, with covering space the upper half plane, $H$, is an example of a "point-pair invariant", i.e., a symmetric kernel function $k(x, y)$, with $x, y$ any two points in some domain $\mathcal{F}$ of arbitrary dimension, such that $k(\gamma[x], \gamma[y])=k(x, y)$ for all $\gamma \in \Gamma$, the covering group of $F$. Then the action of the group induces a symmetric kernel function $\sum_{\gamma \in \Gamma} k(x, \gamma[y])$ in the covering space, $\mathcal{C}$, where $\mathcal{F}=\mathcal{C} / \Gamma$. Selberg's trace formula expresses the kernels of a family of invariant operators, $\{\mathcal{L}\}$, on the domain $\mathcal{F}$ as functions of group invariant objects, $\{\sigma(x, y)\}$. In our example, these are the Laplace-Beltrami operators acting on arbitrary rank tensors on $S$, and there is a single group invariant, $\rho_{H}\left(z, z^{\prime}\right)$, the hyperbolic distance between the two points. The measure for the kernel functions, $\{k(\sigma(x, y))\}$, is the group invariant measure on $\mathcal{C}$, in our example, the $P S L(2, R)$ invariant area element on $H$.

Selberg's trace formula expresses any kernel function on $\mathcal{F}$ as a grouptheoretic trace over a distinguished subset of elements of the covering group $\Gamma$ known as the primitive elements: elements which cannot be expressed as a power of any other element of the group [2]. A crucial role is played in this derivation by the similarity relation of the covering group. To illustrate this, it is helpful to understand a concrete example and we will therefore begin with a review of the domain and primitive elements of the modular group of $S$. But the reader should keep in mind that the applicability of Selberg's method to the computation of symmetric kernel functions is much more general than this specific example illustrates. For an account of some physics applications other than string theory, consult the articles and references cited in the collection [23].

\section{B Uniformization and Kleinian Groups}

Any Riemann surface $S_{(b, c)}^{h}$, with $b$ holes, $c$ crosscaps, and $h$ handles, can be globally uniformized, i.e., represented parametrically by single valued holomorphic or meromorphic functions in a subdomain, $F_{(b, c)}^{h}$, of the Riemann sphere, $\hat{C}$, the universal covering space of $S[11]$. An isometry of the Riemann sphere is an element of the Mobius group, linear fractional transformations on the complex plane, acting on coordinates, $z$, as $\gamma[z]=\frac{a z+b}{c z+d}$, with $a, b, c, d \in \mathbf{C}$, and $a d-b c=1$. The discrete subgroup of Mobius transformations associated 
with the isometries of the subdomain $F$, is a Kleinian subgroup of the Mobius group. A special case is a Fuchsian subgroup, obtained under uniformization to a subdomain of the Poincare upper half plane, $H$. Every compact oriented surface with negative Euler character, $\chi=2-2 h-b$, constant Gaussian curvature, $R_{g}$, and geodesic boundaries, can be globally uniformized to a polygon with $-9 \chi$ sides in $H, F \sim H / \Gamma$, whose shape is parameterized by $-3 \chi$ real moduli parameters. The Gaussian curvature is normalized to $R_{g}=-1$ and, from the Gauss-Bonnet theorem, the area equals a constant, $A=-4 \pi \chi$.

Geodesics in the complex plane with hyperbolic metric are circular arcs degenerating, for sufficiently large radius, to straight lines perpendicular to the real axis. Under a conformal mapping of $H$ to the interior of the unit disk, $D$, the domain $H / \Gamma$ maps to a subdomain of $D$, with homeomorphically related hyperbolic metric. This is an equivalent global uniformization of the Riemann surface. Note that the Kleinian covering groups associated with either choice of global uniformization necessarily coincide, although the subdomains of the complex plane are distinct. One can move freely between these two equivalent choices of domain as convenient [22].

It is possible to give an explicit representation in terms of $\operatorname{PSL}(2, \mathrm{R})$ matrices for the generating elements of the Fuchsian group, $\Gamma_{b}$, of an orientable Riemann surface, $S_{b}$, with $b$ holes and no handles [14]. The conjugacy classes of $\Gamma_{b}$ correspond to homotopically inequivalent classes of closed paths on $S_{b}$. Cutting open $S_{b}$ along $b-3$ interior geodesic paths gives $b-2$ pants: surfaces of constant curvature -1 with three holes, $\mathcal{P}$. Then the generating elements of $\Gamma_{b}$ can be expressed as simple products of the generating elements of the individual $\Gamma_{3}^{(j)}, j=1, \cdots, b-2$, as reviewed in [12][14][15].

A pants surface has three homotopically inequivalent classes of paths, $C[i]$. An arbitrary non-intersecting path on $\mathcal{P}$ is homotopic to one of 3 boundaries, but $C[1] C[2] \sim C[3]$, so that the matrix representatives of the generating elements satisfy the constraint, $\prod_{i=1}^{3} \gamma_{i}=\mathbf{1}$. Consider an arbitrary path on $\mathcal{P}$ joining any two points, $P, P^{\prime}$. Under uniformization to a subdomain of $H$, this maps into a semi-circular arc of length parameter, $|\operatorname{tr} \gamma|$, where $z^{\prime}=\gamma[z]$, and $\gamma$ is an element of the covering group $\Gamma_{3}$. The length parameter coincides with the geodesic length between the points $z, z^{\prime}$, on $H / \Gamma_{3}$ for those linear transformations that act as magnifications. We will be especially interested in geodesic paths. These are singled out by an application of the similarity relation for the covering group: every element of a 
Fuchsian group $\Gamma_{b}$ can be related by a similarity transformation to an orientation preserving magnification, $z^{\prime}=\alpha z$ [12]. The magnifications, $\{\alpha\}$, serve as representative elements of the conjugacy classes of the covering groups, $\Gamma_{b}$.

\section{Fundamental Polygons and Primitive Ele- ments}

Let us understand the meaning of a conjugacy relation for the elements of the fundamental group $\Gamma$ of some domain $\mathcal{F} \sim \mathcal{C} / \Gamma$. As explained above, an arbitrary element in $\Gamma$ can be transformed to a representative element, $\alpha$, by a conjugacy relation, $\beta^{-1} \alpha \beta=\gamma$, for some $\beta \in \Gamma$. The minimum distance along the curve $(z, \alpha[z])$, measured with the group invariant metric, for paths belonging to the conjugacy class of $\alpha$ determines a length parameter. For the hyperbolic metric and the modular group $\Gamma_{b}$, this parameter is:

$$
\begin{aligned}
l(\alpha) & \equiv \operatorname{Min} \operatorname{Cosh}^{-1}\left[1+\frac{1}{2} \frac{|z-\alpha[z]|^{2}}{\alpha(\operatorname{Im} z)^{2}}\right] \\
& =\operatorname{Cosh}^{-1}\left[\frac{1}{2}\left(\alpha+\alpha^{-1}\right)\right],
\end{aligned}
$$

coincident with the trace of the representative group element, $\alpha \in \Gamma$. Thus, the conjugacy class of the element $\alpha$ is isomorphic to a deformation class of geodesic paths: paths homotopically equivalent to $\alpha\left[z_{0}\right]$, defined with respect to some base point $z_{0}$ that varies freely in $H / \Gamma_{b}$. The length parameter for repeated magnifications is additive, giving the relation, $l\left(\alpha^{n}\right)=|n| l(\alpha)$, for every $n \in Z[8]$. These comments can be generalized to the general case.

This suggests a natural prescription for performing the sum over conjugacy classes of a covering group $\Gamma$. We begin by defining a primitive element, $\delta$ : a group element which cannot be expressed as a power of any other element in $\Gamma$. From the relation above, an arbitrary magnification, $\alpha \in \Gamma$, can be expressed as a power, $n$, of a primitive magnification, $\alpha=\delta^{n}$. Arbitrary group elements, $\gamma$, are then obtained by a sum over elements in the conjugacy class of $\alpha$ : $\gamma=\beta^{-1} \delta^{n} \beta, \forall \beta \in \Gamma$. This would overcount by an overall infinity due to elements in $\Gamma$ that commute with $\delta$, since for any such element the conjugacy relation maps $\delta^{n}$ into itself. Let us therefore define the 
centralizer of the primitive element $\delta, \Gamma^{(\delta)}$, as the set of elements in $\Gamma$ that commute with $\delta$. Running once through the primitive magnifications, $\delta$, and then summing, for fixed $\delta$, over every element $\beta \in \Gamma / \Gamma^{(\delta)}$, runs exactly once through the conjugacy classes of the group, $\Gamma$. The conjugacy class of any primitive magnification is therefore the set:

$$
\{\delta\} \equiv\left\{\gamma \mid \gamma=\left(\beta^{-1} \delta^{n} \beta\right) \forall \beta \in \Gamma / \Gamma^{(\delta)}, n \in \mathrm{Z}^{+}\right\} .
$$

Thus, a sum over the conjugacy classes of the covering group can be written as the nested sum:

$$
\sum_{\{\delta\}}=\sum_{\delta} \sum_{\beta \in \Gamma / \Gamma^{(\delta)}} \sum_{n=1}^{\infty} .
$$

This identity can be used to relate the Greens function on the covering space $H$ to that on the fundamental polygon, $H / \Gamma_{b}$. This analysis of primitive elements for a Fuchsian group straightforwardly generalizes to the loxodromic Kleinian groups associated to a nonorientable surface [11]. It should be noted that Selberg's formalism can be applied to the Schottky covering group of $S$, under global uniformization to a disjoint subdomain of the flat complex plane: the fundamental domain of a genus $h$ closed Riemann surface is the region in the complex plane exterior to $2 h$ isometric circles, $C_{j}, C_{j}^{\prime}, j=1, \cdots, h$. However, the decomposition into primitive elements could get involved.

\section{Derivation of the Trace Formula}

The analysis of conjugacy classes of the Fuchsian group $\Gamma_{b}$ applies to any covering group for which we can identify primitive elements. For such a group, the sum over conjugacy classes, $\sum_{\{\delta\}}$, can be written as the nested sum:

$$
\sum_{\{\delta\}} \int_{\mathcal{C} / \Gamma} d x k(x, \gamma[x])=\sum_{\delta} \sum_{\beta \in \Gamma / \Gamma^{(\delta)}} \sum_{n=1}^{\infty} \int_{\mathcal{C} / \Gamma} d x k\left(x,\left(\beta^{-1} \delta^{n} \beta\right)[x]\right)
$$

where $d x$ denotes the group invariant measure in the domain $\mathcal{F} \sim \mathcal{C} / \Gamma$. Extracting the identity element from the sum over primitive elements, we can simplify:

$$
\sum_{\{\delta\}} \int_{\mathcal{C} / \Gamma} d x k(x, \gamma[x])=\sum_{\delta \neq \mathbf{1}} \sum_{n=1}^{\infty} \sum_{\beta \in \Gamma / \Gamma^{(\delta)}} \int_{\beta[\mathcal{C} / \Gamma]} d x k_{0}\left(x, \delta^{n}[x]\right)+\int_{\mathcal{C} / \Gamma} d x k_{0}(x, x),
$$


where $\beta[\mathcal{C} / \Gamma]$ denotes the image of the fundamental domain under the action of the group element, $\beta$. The result is therefore expressed as a sum over kernels $k_{0}\left(x, \delta^{n}[x]\right)$, each of which is integrated over the domain, $\mathcal{C} / \Gamma^{(\delta)}$, of an inconjugate primitive element $\delta$ :

$$
\sum_{\delta \neq \mathbf{1}} \sum_{n=1}^{\infty} \int_{\mathcal{C} / \Gamma_{\delta}} d x k_{0}\left(x, \delta^{n}[x]\right)+\int_{\mathcal{C} / \Gamma} d x k_{0}(x, x)
$$

Here, $k_{0}(x, \gamma[x])$ is the kernel function induced on $\mathcal{C}$ by the action of the covering group. Note that this mapping is bijective so we can interchange the roles of $k(x, y)$ and $k_{0}(x, y)$, obtaining either one from the other, given an explicit parameterization of $\Gamma$. The second term in this equation is simply the area of the domain multiplied by the kernel function with coincident arguments: $\operatorname{Area}(\mathcal{C} / \Gamma) k_{0}(\sigma=0)$. This is the Selberg trace formula [2].

\section{E Length Spectrum and Poincare Theta Se- ries on $S_{b}$}

For the Fuchsian groups, $\Gamma_{b}$, the Selberg formula can be simplified as follows. Consider a magnification, $\delta^{p}[z]=e^{p l(\delta)} z$. Then,

$$
\operatorname{tr}^{\prime} e^{-\left(\Delta+m^{2}\right) t}=\sum_{\delta \neq \mathbf{1}} \sum_{p=1}^{\infty} \int_{H / \Gamma^{(\delta)}} \frac{d x d y}{y^{2}} \hat{G}\left(z, e^{p l(\delta[z])} ; t\right)+A \cdot \hat{G}(z, z ; t) \quad,
$$

where the argument of $\hat{G}\left(z, z^{\prime} ; t\right)$ is the hyperbolic distance between $z, z^{\prime}$ in $H / \Gamma_{b}$ :

$$
\operatorname{Cosh}^{2}\left(\rho_{H}\left(z, e^{p l(\delta) / 2)} z\right)\right)=1+\left(1+x^{2} / y^{2}\right) \operatorname{Sinh}^{2}\left(\frac{1}{2} p l(\delta)\right),
$$

where the contribution from the identity element to the group theoretic trace has been singled out. Here, $A=-4 \pi \chi$. A change of variables, $(x, y) \rightarrow\left(u=x / y, l^{\prime}=\ln y\right)$, in the trace formula gives the result:

$$
\operatorname{tr}^{\prime} e^{-\left(\Delta+m^{2}\right) t}=\sum_{\delta \neq \mathbf{1}} \sum_{p=1}^{\infty} \int_{-\infty}^{\infty} d u\left[\frac{\sqrt{2} l(\delta)}{(4 \pi t)^{3 / 2}} \int_{\rho_{H}}^{\infty} d b \frac{b e^{-b^{2} / 4 t-M^{2} t}}{\sqrt{\operatorname{Cosh} b-\operatorname{Cosh} \rho_{H}}}\right],
$$

where we have suppressed the contribution to the trace from the identity

element: $A \cdot \hat{G}\left(\operatorname{Cosh}\left(l_{\min .}\right) ; t\right)$, the area times the Greens function at the 
source. In the absence of an ultraviolet cutoff on the world-sheet, we can simply replace $l_{\min }$. with zero but it should be kept in mind that $l_{\min }$. is really of $O\left(\alpha^{\prime} / r^{2}\right)$, world-sheet ultraviolet regulator, $\epsilon$, dependent.

Interchanging the orders of integration over $b, u$, the half-space, $b \geq \rho_{H}$, with $u$ arbitrary, can be mapped to the half-strip, $-u_{0} \leq u \leq u_{0}, b \geq$ $p l(\delta)$, where $u_{0}=[\operatorname{Cosh}(b)-\operatorname{Cosh}(p l(\delta))]^{1 / 2} / \sqrt{2} \operatorname{Sinh}(p l(\delta)) / 2$. Performing the integrations gives the Poincare theta series:

$$
\Theta(t)=\left[\sum_{\delta \neq \mathbf{1}} \sum_{p=1}^{\infty} \frac{l(\delta) / 2}{\operatorname{Sinh}(p l(\delta) / 2)} e^{-p^{2} l^{2} / 4 t}\right] \frac{e^{-M^{2} t}}{(\pi t)^{1 / 2}} .
$$

\section{F Selberg Zeta Function}

Selberg's trace formula can be converted to infinite product form, yielding the Selberg zeta functions with properties similar to those of the Jacobi theta functions. These are especially helpful in examining the large and small $l(\delta)$ asymptotics. A Laplace transform of the Poincare theta series, $\Theta(t)$, is an entire function in the $s$ plane and, in particular, is regular at the origin, $s=0$ [8]. Consider the logarithmic derivative of $Z(s)$ with respect to the variable $s$. Then,

$$
\frac{Z^{\prime}(s)}{Z(s)} \equiv\left(s-\frac{1}{2}\right) \int_{0}^{\infty} e^{-s(s-1) t} \Theta(t) d t \quad,
$$

which can be considered as a functional equation whose solution is the Selberg zeta function, $Z(s)$, defined as:

$$
Z(s)=\prod_{\delta \neq \mathbf{1}} \prod_{k=0}^{\infty}\left[1-e^{-(s+k) l(\delta)}\right] .
$$

It is easy to verify that this definition is consistent with the integral transform of $\Theta(t)$. We begin with the left-hand-side:

$$
\begin{aligned}
\frac{Z^{\prime}(s)}{Z(s)} & =\sum_{\delta \neq \mathbf{1}} \sum_{k=0}^{\infty} l(\delta) \frac{e^{-(s+k) l(\delta)}}{1-e^{-(s+k) l(\delta)}} \\
& =\sum_{\delta \neq \mathbf{1}} l(\delta) \sum_{k=0}^{\infty} \sum_{n=1}^{\infty} e^{-n(s+k) l(\delta)}
\end{aligned}
$$




$$
=\sum_{p=1}^{\infty} \sum_{\delta \neq \mathbf{1}} \frac{l(\delta) / 2}{\operatorname{Sinh}\left(\frac{1}{2} l\left(\delta^{p}\right)\right)} e^{-\left(s-\frac{1}{2}\right) l\left(\delta^{p}\right)},
$$

where we have used $l\left(\delta^{p}\right)=p l(\delta)$. We recognize the argument of the sum as $\Theta(t)$ by making use of the following identity for Gaussian integrals:

$$
e^{-\left(s-\frac{1}{2}\right) l\left(\delta^{p}\right)}=\left(s-\frac{1}{2}\right) \int_{0}^{\infty} \frac{d t}{(4 \pi t)^{1 / 2}} e^{-\left(s-\frac{1}{2}\right)^{2} t-\left|l\left(\delta^{p}\right)\right|^{2} / 4 t}
$$

Direct substitution yields the functional relation above:

$$
\frac{Z^{\prime}(s)}{Z(s)}=\left(s-\frac{1}{2}\right) \sum_{p=1}^{\infty} \sum_{\delta \neq \mathbf{1}} \frac{2 l(\delta)}{\operatorname{Sinh}\left(\frac{1}{2} l\left(\delta^{p}\right)\right)} \int_{0}^{\infty} \frac{d t}{(4 \pi t)^{1 / 2}} e^{-|p l(\delta)|^{2} / 4 t} e^{-t / 4-s(s-1) t} .
$$

\section{References}

[1] J. Polchinski, String Theory, Volumes I and II (Cambridge). See, in particular, section 9.5 for a clear statement on finiteness in string theory.

[2] A. Selberg, Harmonic Analysis and Discontinuous Groups in Weakly Symmetric Riemannian Spaces With Applications to Dirichlet Series, J. Indian Math. Soc. Vol. 20, 47 (1956).

[3] J. Polchinski, Evaluation of the One Loop String Path Integral, Comm. Math. Physics, 104, 37.

[4] E. D'Hoker and D.H. Phong, Multiloop Amplitudes for the Bosonic Polyakov String, Nucl. Phys. B269 (1986) 205.

[5] G. Frye and L. Susskind, Non-planar Dual Symmetric Loop Graphs and the Pomeron, Phys. Lett. B31 (1970) 589. H. Nielsen, NORDITA preprint (1969); D. Fairlie and H. Nielsen, Nucl. Phys. B20 (1970) 637. C. Lovelace, M loop Generalized Veneziano Formula, Phys. Lett. B32 (1970) 703. V. Alessandrini, Generalized Approach to Dual Multiloop Dia grams, Il. Nuovo Cimento Vol. 2A (1971) 321; V. Alessandrini and D. Amati, Properties of Dual Multiloop Amplitudes, Il. Nuovo Cimiento Vol. 4A (1971) 793. 
[6] C. Bachas, Dbrane Dynamics, Phys. Lett. B374 (1996) 37, hepth/9511043.

[7] M. Douglas, D. Kabat, P. Pouliot, and S. Shenker, Dbranes and Short Distances in String Theory, Nucl. Phys. B485 (1997) 85, hepth/9608024.

[8] H. P. McKean, Selberg's Trace Formula As Applied to a Compact Riemann Surface, Comm. Pure \& Applied Math., Vol. XXV, 225 (1972).

[9] O. Alvarez, Theory of Strings with Boundaries: Fluctuations, Topology, and Quantum Geometry, Nucl. Phys. B216 (1983) 125.

[10] V. Periwal and O. Tafjord, Finite Cutoff on the String Worldsheet?, hep-th/9803195.

[11] L. Bers, Uniformization, Moduli, and Kleinian Groups, Bull. London Math. Soc. 4 (1972) 257.

[12] W. Abikov, The Real Analytic Theory of Teichmuller Space, Lecture Notes in Mathematics, 820, Springer-Verlag.

[13] R. Fricke and F. Klein, Vorlesungen uber die Theorie der Automorphen Functionen, Tuebner, Leipzig, 1896/1912.

[14] L. Keen, On Fricke Moduli, Annals Math. Studies, Vol. 66 (1971) 205.

[15] S. Wolpert, The length spectra as moduli for compact Riemann surfaces, Ann. Math. 169 (1969) 323.

[16] See the recent review, J. Polchinski, M-Theory and the Light Cone, hepth/9903165, and references within.

[17] W. Fenchel and J. Nielsen, Discontinuous Groups of Non-Euclidean Motions, unpublished manuscript.

[18] A. M. Polyakov, Quantum Geometry of Bosonic Strings, Phys. Lett. B103 (1981) 207.

[19] A. Fischer and A. J. Tromba, On the Weil-Petersson Metric on Teichmuller Space, Trans. AMS, Volume 284, 1 (1984) 319. 
[20] S. Wolpert, The Fenchel-Nielsen Deformation, Ann. Math. 115 (1982) 501.

[21] J. Maldacena, Wilson Loops in large N Field Theories, hep-th/9803002; see, also, the recent paper, N. Drukker, D.J. Gross, and H. Ooguri, Wilson Loops and Minimal Surfaces, hep-th/9904191.

[22] See, for example, A. Terras, Harmonic Analysis on Symmetric Spaces and Applications, Volume I, Chap. 3 (Springer Verlag).

[23] D. Hejhal, The Selberg Trace Formula, Lect. Notes in Math. 47. D. Hejhal ed., The Selberg Trace Formula and Related Topics, proceedings of the AMS-IMS-SIAM Joint Summer Conference, July 1984 (c1986). Proceeedings of the 1997 NATO ASI on Supersymmetry and Trace Formulae: Chaos and Disorder, eds. T.V. Lerner, J. Keating, and D. Khmelnitski, Plenum (c1999).

[24] H. Huber, Math. Annals, Vol 138, 1959, 1-26.

[25] S. Wolpert, Asymptotics of the Spectrum and the Selberg Zeta Function on the Space of Riemann Surfaces, Comm. Math. Phys. 112 (1987) 283, and references within.

[26] P. Nelson, Lectures on Strings and Moduli Space, Phys. Rep. 149 (1987) 337.

[27] S. Hawking, Zeta Function Regularization of Path Integrals in Curved Spacetime, Comm. Math. Phys. 55133 (1977).

[28] S. Chaudhuri, Path Integral Evaluation of Dbrane Amplitudes, PSU preprint PSU-TH-206, hep-th/9903184.

[29] S. Chaudhuri and Y. Chen, in preparation.

[30] J. Fay, Fourier Coefficients of the Resolvent for a Fuchsian Group, J. Reine Angew. Math. 293, 143 (1977).

[31] E. D'Hoker and D.H. Phong, Length-Twist Parameters in String Path Integrals, Phys. Rev. Lett. 56 (1986) 912. 
[32] See T. Branson, P. Gilkey, K. Kirsten, and D. Vassilevich, Heat Kernel Asymptotics with mixed boundary conditions, hep-th/9906144, and references within.

[33] See the recent paper, J. Ambjorn, Yu. Makeenko, G.W. Semenoff, and R. J. Szabo, Screening and Dbrane Dynamics in Finite Temperature Superstring Theory, hep-th/9906134.

[34] J. Maldacena, The Large N limit of Superconformal Field Theories and Supergravity, hep-th/9711200.

[35] N. Itzhaki, J. Maldacena, J. Sonnenschein, and S. Yankielowicz, Supergravity and the Large $N$ limit of Theories with Sixteen Supercharges, hep-th/9802042.

[36] A. Hashimoto and N. Itzhaki, A Comment on the Zamolodchikov cfunction and the Black String Entropy, hep-th/9903067; F. Antonuccio, A. Hashimoto, O. Lunin, and S. Pinsky, Can DLCQ test the Maldacena conjecture?, hep-th/9906087.

[37] S. Chaudhuri, The Normalization of Perturbative String Amplitudes: Weyl Covariance and Zeta Function Regularization, pedagogical electronic review, hep-th/0409031.

[38] S. Chaudhuri, Thermal Duality Transformations and the Canonical Ensemble: The Deconfining Long String Phase Transition, hep-th/0409301.

[39] S. Chaudhuri, Microcanonical Ensemble of Type I Strings, in preparation. 